Journal of Engineering and Applied Sciences 14 (11): 3611-3614, 2019

ISSN: 1816-949X

(C) Medwell Journals, 2019

\title{
Sumrate Maximization by Optimal Precoder Design for MIMO Retransmission System
}

\author{
K. Sakthidasan Sankaran, V. Beslin Geo, S.M. Umarani and P. Archana \\ Hindustan Institute of Technology and Science, \\ Department of Electronics and Communication Engineering, 603103 Chennai, India
}

\begin{abstract}
MIMO system offers substantial gain in through put and link reliability in wireless systems. Precoding in MIMO plays a vital role in mitigating fading and co-channel interference effects in time-variant channels. Despite of these advantages, the wireless system is more prone to packet errors and loss due to channel distortions, noise or fading. This in turn invokes the necessity of retransmission in MIMO. A codebook based precoding approach is used in retransmission systems in which instead of using a single codebook, a new codebook is designed for each retransmission using Lloyd algorithm which minimizes the average distortion. This approach combines the effect of precoding and retransmission in MIMO thereby providing high sum rate and BER.
\end{abstract}

Key words: $\mathrm{MIMO}$, CSI, precoding, codebook, retransmission, retransmission

\section{INTRODUCTION}

Recent advancements in wireless systems have led to the performance gains and increase in demand of scarce spectrum. Multiple Input Multiple Output (MIMO) system achieves effective usage of spectrum through spatial multiplexing technique. MIMO along with precoding alleviates the problem of interference and fading due to the time varying nature of channels. Precoding combats these effects by using Channel State Information (CSI) available at the transmitter. In Frequency Division Duplex (FDD) systems, the CSI is acquired through a limited feedback channel from receiver to transmitter. To reduce the feedback overhead, a codebook based precoding approach is used in which the receiver selects a precoding matrix from the codebook based on some criterion and the Precoding Matrix Index (PMI) will be fed back to the transmitter. Despite of the benefits delivered by the MIMO links, the packet loss and errors in the channels necessitates retransmission. To further improve the link reliability, MIMO systems are assisted with Automatic Repeat Request (ARQ) mechanism in which packets which fails to provide satisfactory performance will be retransmitted. MIMO systems integrated with precoding and ARQ mechanism will enhance sum rate and BER.

Different codebook based precoding techniques are discussed by Yang et al. (2012), Jiang et al. (2011) Xiao et al. (2012), Kim et al. (2011) and Song and Lee
(2008). By Yang et al. (2012), the researcher proposes a codebook-based LRP (Lattice Reduction Precoding) scheme for limited-feedback coded MIMO systems. It employs multilevel binary coset coding, so that, the precoding matrix is binary which is computed offline. To reduce the feedback bits and to improve the size of the codebook a rotating codebook design technique is proposed (Jiang et al., 2011). And to reduce the storage requirements of codebook at the transmitter and receiver, a structured block-circulant codebook approach is used in which the given first codeword, rotation matrix and other codewords are found (Xiao et al., 2012). To further enhance the performance, adaptive precoding is performed by adding the perturbation vector which is determined by the directional variation to the previously used precoder (Kim et al., 2011). Codebook is designed online by using generalized Lloyd algorithm in which centroid computation is considered as an optimization problem (Song and Lee, 2008).

To enhance the sum rate capacity and BER MIMO systems are integrated with precoding and retransmission. By Sun et al. (2006), the researcher derives a sequence of successive optimal ARQ precoders which minimizes the MSE (Mean Square Error) between the transmitted data and the joint receiver output but it requires full CSI. Along with minimizing MSE in the retransmission system, the proposed system aims to cancel the interference effectively by using the block decision feedback detection under the assumption of perfect

Corresponding Author: K. Sakthidasan Sankaran, Hindustan Institute of Technology and Science, 
feedback (Sun and Ding, 2007). By Kim and Kang (2011), sequential linear precoder is designed to maximize the mutual information by using gradient search algorithm in MIMO hybrid ARQ systems.

\section{MATERIALS AND METHODS}

A CL-MIMO (Closed Loop-MIMO) system with $\mathrm{N}_{\mathrm{t}}$ transmit antennas and $\mathrm{N}_{\mathrm{r}}$ receive antennas are considered. Let's be the $M \times 1$ transmit symbol vector for which is to be coded using $\mathrm{N}_{\mathrm{t}} \times \mathrm{M}$ precoding matrix $\mathrm{F}$. Then the precoded transmit symbol vector must be transmitted over $\mathrm{N}_{\mathrm{r}} \times \mathrm{N}_{\mathrm{t}}$ channel matrix $\mathrm{H}$. To guarantee symbol recoverability it is assumed that $\mathrm{MN}_{\mathrm{r}}$. For retransmission MIMO system, different precoding matrices must be used for each transmission. If a total of $m$ transmissions are considered then the received signal vector $Y_{m}$ after the $\mathrm{mth}$ transmission is given by:

Where:

$$
\mathrm{Y}_{\mathrm{m}}=\mathrm{H}_{\mathrm{m}} \mathrm{F}_{\mathrm{m}} \mathrm{s}+\mathrm{n}_{\mathrm{m}}
$$

$\mathrm{H}_{\mathrm{m}}$ and $\mathrm{F}_{\mathrm{m}}=$ The channel matrix and precoding matrix of mth transmission, respectively

$\mathrm{n}_{\mathrm{m}} \quad=$ The zero mean complex Gaussian noise vector (Fig. 1)

The concatenated received signal vector $\overline{\mathrm{Y}}_{\mathrm{m}}$ after $\mathrm{m}$ transmissions and receptions is given by:

$$
\overline{\mathrm{Y}}_{\mathrm{m}}=\left[\begin{array}{c}
\mathrm{Y}_{1} \\
\vdots \\
\mathrm{Y}_{\mathrm{m}}
\end{array}\right]=\left[\begin{array}{c}
\mathrm{H}_{1} \mathrm{~F}_{1} \\
\vdots \\
\mathrm{H}_{\mathrm{m}} \mathrm{F}_{\mathrm{m}}
\end{array}\right] \mathrm{s}+\left[\begin{array}{c}
\mathrm{n}_{1} \\
\vdots \\
\mathrm{n}_{\mathrm{m}}
\end{array}\right]=\overline{\mathrm{H}}_{\mathrm{m}} \mathrm{s}+\overline{\mathrm{n}}_{\mathrm{s}}
$$

where, $\overline{\mathrm{H}}_{\mathrm{m}}$ and $\overline{\mathrm{n}}_{\mathrm{m}}$ are the concatenated channel and noise matrices, respectively. A linear detection scheme is used after $m$ transmissions and the detected symbol vecto $\hat{\mathrm{s}}$ is given by:

$$
\hat{\mathrm{s}}=\mathrm{G}_{\mathrm{m}} \overline{\mathrm{Y}}_{\mathrm{m}}=\mathrm{G}_{\mathrm{m}} \overline{\mathrm{H}}_{\mathrm{m}} \mathrm{s}+\overline{\mathrm{n}}_{\mathrm{m}}
$$

where, $G_{m}$ is the linear detection matrix which is based on the Minimum Mean Square Error (MMSE) criterion.

Codebook design: Precoding is done effectively by using codebook. It consists of a set of precoding matrices which is constructed offline or online from which a precoding matrix will be selected based upon some desired criterion to improve the performance of the system. The effectiveness of a codebook lies in the selection of optimal precoding matrix with minimum number of feedback bits. The choice of optimal precoding matrix lies

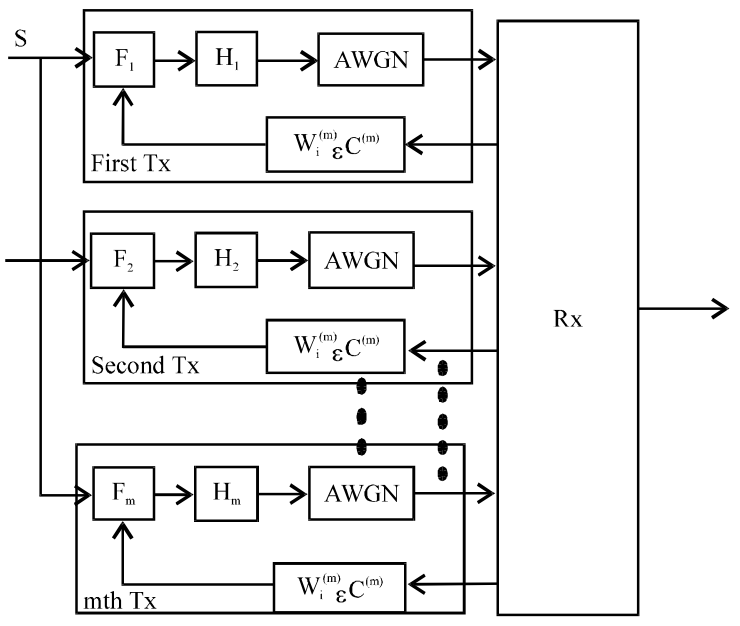

Fig. 1: System model of codebook based MIMO retransmission system

in the codebook design methods and selection criteria. The selection criteria used can be aimed at minimizing the mean square error or maximizing the capacity. Consider a Codebook $\mathrm{C}^{(\mathrm{m})}$ which consists of $\mathrm{n}$ number of precoding matrices such that $C^{(m)}=\left\{F_{1}, F_{2}, \ldots, F_{n}\right\}$. The precoding matrix which minimizes the minimum mean square error can be selected as:

$$
\mathrm{F}=\underset{\mathrm{W}_{\mathrm{i}} \in \mathrm{C}}{\operatorname{argmin}} \operatorname{Tr}\left\{\left(\mathrm{I}_{\mathrm{N}_{\mathrm{t}}}+\gamma\left[\mathrm{HW}_{\mathrm{i}}\right]^{\mathrm{H}}\left[\mathrm{HW}_{\mathrm{i}}\right]\right)^{-1}\right\}
$$

The precoding matrix which maximizes the sum rate capacity can be selected from the Codebook $\mathrm{C}$ as:

$$
\mathrm{F}=\underset{\mathrm{W} \in \mathrm{C}}{\arg \max } \log _{2}\left[\operatorname{det}\left(\mathrm{I}+\frac{\mathrm{E}_{\mathrm{b}}}{\mathrm{N}_{\mathrm{o}} \mathrm{N}_{\mathrm{t}}} \mathrm{H}^{*} \mathrm{~W}^{*} \mathrm{~W}^{\mathrm{H}} * \mathrm{H}^{\mathrm{H}}\right)\right]
$$

The codebook for first transmission can be designed using any of the design methods such as DFT (Discrete Fourier Transform). If the initial transmission fails to give satisfactory performance then the retransmissions are carried out with a new codebook design using Lloyd algorithm.

Retransmission using Lloyd algorithm: The codebook for retransmission is designed using Lloyd algorithm and the optimal precoding matrix which minimizes the mean square error or maximizing the sum rate capacity is selected. It makes use of the channel matrices and precoding matrices of previous retransmissions to design the new codebook. The codebook design steps are as follows. For the mth transmission, an arbitrary codebook 
$\left\{\mathrm{C}^{(\mathrm{m})}\right\}$ is generated as the initial codebook. Then a sequence of channel matrices $\mathrm{H}_{1}, \mathrm{H}_{2}, \ldots, \mathrm{H}_{\mathrm{m}}$ are generated and the corresponding sequence of precoding matrices $F_{1}, F_{2}, \ldots, F_{m-1}$ which reduces the MMSE are generated using:

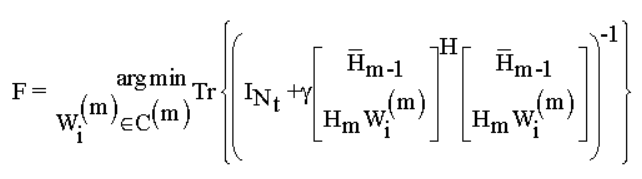

Then the optimal precoding matrix $\mathrm{F}_{\mathrm{m}}^{\text {opt }}$ is generated using the following steps: SVD decomposition of $\mathrm{H}_{\mathrm{m}}$ into:

$$
\mathrm{H}_{\mathrm{m}}=\mathrm{U}_{\mathrm{m}} \Sigma_{\mathrm{m}} \mathrm{V}_{\mathrm{m}}^{\mathrm{H}}
$$

where, $\mathrm{U}_{\mathrm{m}}$ and $\mathrm{V}_{\mathrm{m}}$ are left and right singular matrices and ${ }_{s_{m}}$ is diagonal matrix whose diagonal elements are the singular values of $\mathrm{H}_{\mathrm{m}}$. Decomposition of Hermitian matrix into:

$$
\sum_{\mathrm{i}=1}^{\mathrm{m}-1} \mathrm{~F}_{\mathrm{i}}^{\mathrm{H}} \mathrm{H}_{\mathrm{i}}^{\mathrm{H}} \mathrm{H}_{\mathrm{i}} \mathrm{F}_{\mathrm{i}}=\mathrm{U}_{\mathrm{m}-1} \Lambda_{\mathrm{m}-1} \mathrm{U}_{\mathrm{m}-1}^{\mathrm{H}}
$$

Then, the optimal precoding matrix is given by:

$$
\mathrm{F}_{\mathrm{m}}^{\mathrm{opt}}=\overline{\mathrm{V}}_{\mathrm{m}} \mathrm{P} \overline{\mathrm{U}}_{\mathrm{m}-1}^{\mathrm{H}}
$$

where, $\mathrm{P}$ is the permutation matrix obtained by rearranging the order of the diagonal entries in ${ }_{r_{m}-1}$ and $\varepsilon_{m}$ in reverse order. The $\overline{\mathrm{v}}_{\mathrm{m}}$ and $\overline{\mathrm{U}}_{\mathrm{m}-1}$ are obtained from first $\mathrm{M}$ columns of $\mathrm{V}_{\mathrm{m}}$ and $\mathrm{U}_{\mathrm{m}}$, respectively. After generating an optimal precoding matrix a training set of the un-quantized optimum precoding matrices $\left\{\mathrm{F}_{\mathrm{m}}{ }^{\mathrm{opt}}\right\}$ is generated. The partition cells $\left\{\mathrm{R}_{\mathrm{i}}\right\}$ are constructed using the current codebook $\left\{\mathrm{C}^{(\mathrm{m})}\right\}$ and the training set $\left\{\mathrm{F}_{\mathrm{m}}^{\mathrm{opt}}\right\}$ based on the Nearest Neighborhood Condition (NNC) given by:

$$
\mathrm{R}_{\mathrm{i}}=\left\{\mathrm{F} \mid \mathrm{d}\left(\mathrm{F}, \mathrm{W}_{\mathrm{i}}^{(\mathrm{m})}\right)<\mathrm{d}\left(\mathrm{F}, \mathrm{W}_{\mathrm{j}}^{(\mathrm{m})}\right), \forall \mathrm{j} \neq \mathrm{i}\right\}, \mathrm{F} \in\left\{\mathrm{F}_{\mathrm{m}}^{\mathrm{opt}}\right\}(10)
$$

The distance metric used is chordal distance and is given by:

$$
\mathrm{d}(\mathrm{A}, \mathrm{B})=\frac{1}{\sqrt{2}}\left\|\mathrm{AA}^{\mathrm{H}}-\mathrm{BB}^{\mathrm{H}}\right\|_{\mathrm{F}}
$$

Centroid in each partition cell $\overline{\mathrm{W}}_{\mathrm{i}}^{(\mathrm{m})}$ is calculated using Centroid Condition (CC) which minimizes the distance between $\mathrm{F}_{\mathrm{m}}^{\text {opt }}$ and $\mathrm{W}_{\mathrm{i}}^{(\mathrm{m})}{ }_{\text {ds }}\left\{\mathrm{R}_{\mathrm{i}}\right\}$. Then each codeword matrix must be replaced by the centroid of its partition cell, i.e., $\mathrm{W}_{\mathrm{i}}^{(\mathrm{m})} \leftarrow \overline{\mathrm{W}}_{\mathrm{i}}^{(\mathrm{m})}$. Iterate the $\mathrm{NNC}$ and $\mathrm{CC}$ condition until the convergence of optimal codebook occurs. Then, retransmit using the new codebook by using Lloyd algorithm, the sum rate maximization and reduction of BER can be achieved in MIMO retransmission system.

\section{RESULTS AND DISCUSSION}

In this study, the performance gains achieved by using Lloyd algorithm in MIMO retransmission systems are discussed. The simulation results are compared for different order MIMO systems and different modulation systems. Figure 2 illustrates the sum rate capacity of different order MIMO systems. It is clear that the sum rate capacity logarithmically increases when the number of antenna gets increased. Figure 3 depicts the bit error probability of different modulation systems using Lloyd algorithm based precoding technique. The BPSK and QPSK modulation achieves low BER at low SNR compared to 16-QAM system. Figure 4 illustrates the BER for

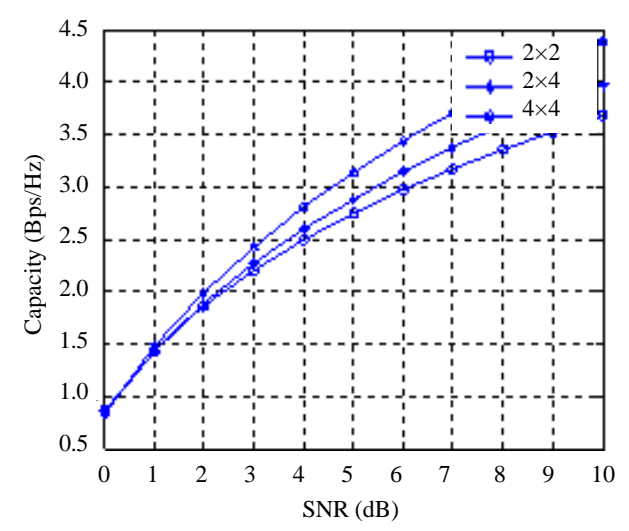

Fig. 2: Sum rate capacity of different order MIMO retransmission system

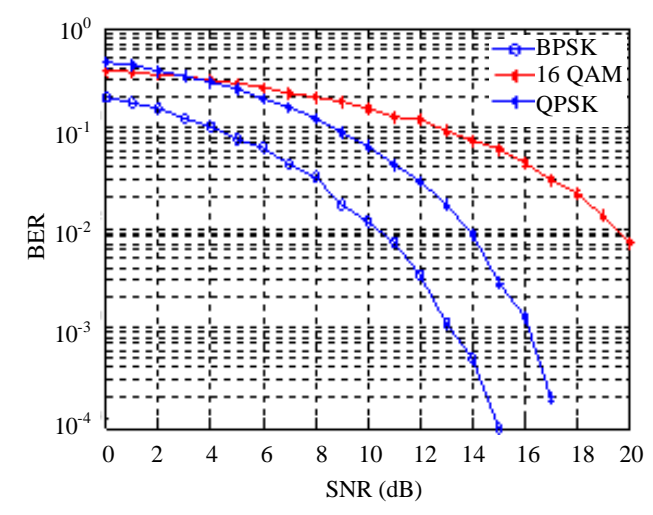

Fig. 3: Bit error probabilty curve of different modulation systems 


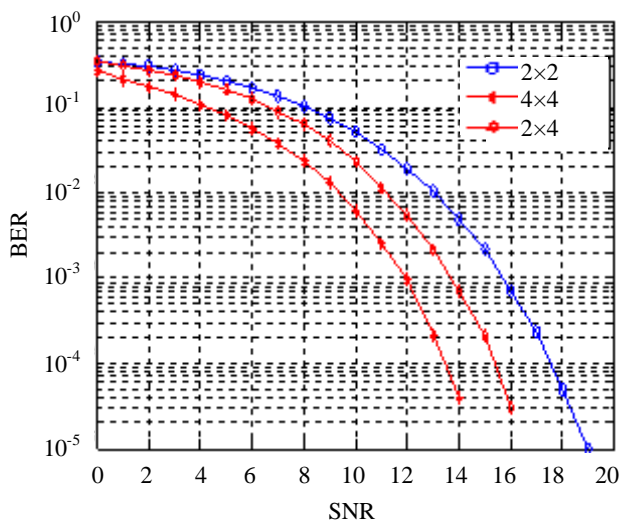

Fig. 4: BER curve of different order MIMO retransmission system

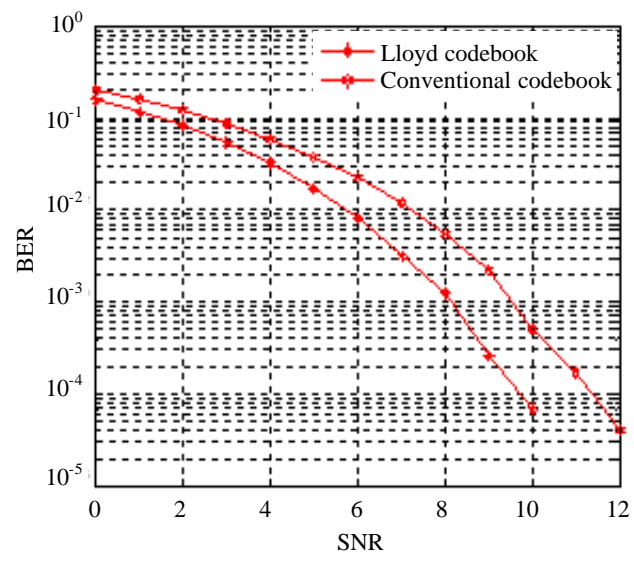

Fig. 5: Comparison between BER curve of conventional vs. Lloyd algorithm based precoding in MIMO retransmission system

different order MIMO systems with QPSK modulation. When the number of antenna increases, BER also get improved. Figure 5 compares the BER of conventional codebook based retransmission structure and Lloyd algorithm based retransmission structure. It is obvious that Lloyd algorithm based retransmission outperforms codebook based retransmission at an SNR of $10 \mathrm{~dB}$.

\section{CONCLUSION}

In this study, an optimal linear precoding scheme based on codebook design using Lloyd algorithm in
MIMO retransmission system is considered. This approach uses limited feedback channel and different codebooks for each transmission to enhance the performance. Hence, this method outperforms the precoding scheme where a single codebook is used for all the transmissions. From simulation results it is clear that this method achieves high sum rate capacity and low BER.

\section{ACKNOWLEDGEMENT}

We would like to show our gratitude to our Management of Hindustan Institute of Technology and Science, Chennai for providing insight and expertise that greatly assisted this research.

\section{REFERENCES}

Jiang, C., M.M. Wang, C. Yang, F. Shu and J. Wang et al., 2011. MIMO precoding using rotating codebooks. IEEE. Trans. Veh. Technol., 60: 1222-1227.

Kim, J. and C.G. Kang, 2011. Codebook design for MIMO retransmission system. IEEE. Commun Lett., 15: 953-955.

Kim, T., D.J. Love and B. Clerckx, 2011. MIMO systems with limited rate differential feedback in slowly varying channels. IEEE. Trans. Commun., 59: 1175-1189.

Song, X. and H.N. Lee, 2008. Multimode precoding for MIMO systems: Performance bounds and limited feedback codebook design. IEEE. Trans. Signal Process., 56: 5296-5301.

Sun, H. and Z. Ding, 2007. Iterative transceiver design for MIMO ARQ retransmissions with decision feedback detection. IEEE. Trans. Signal Process., 55: 3405-3416.

Sun, H., J.H. Manton and Z. Ding, 2006. Progressive linear precoder optimization for MIMO packet retransmissions. IEEE. J. Sel. Areas Commun., 24: 448-456

Xiao, P., R. Tafazolli, K. Moessner and A. Gluhak, 2012. Codebook based single-user MIMO system design with widely linear processing. IEEE. Trans. Commun., 60: 1-7.

Yang, H.J., J. Chun, Y. Choi, S. Kim and A. Paulraj, 2012. Codebook-based lattice-reduction-aided precoding for limited-feedback coded MIMO systems. IEEE. Trans. Commun., 60: 510-524. 